\title{
Are We All Scientific Experts Now?
}

Thomas Vogt

Citation: Physics Today 68, 5, 52 (2015); doi: 10.1063/PT.3.2788

View online: https://doi.org/10.1063/PT.3.2788

View Table of Contents: http://physicstoday.scitation.org/toc/pto/68/5

Published by the American Institute of Physics

\section{Articles you may be interested in}

Albert Einstein as a Philosopher of Science

Physics Today 58, 34 (2005); 10.1063/1.2169442

Commentary: Surviving scientist burnout

Physics Today 70, 10 (2017); 10.1063/PT.3.3675

Conductors, Semiconductors, Superconductors: An Introduction to Solid State Physics Physics Today 68, 52 (2015); 10.1063/PT.3.2787

Einstein's Mistakes

Physics Today 58, 31 (2005); 10.1063/1.2155755

The image of scientists in The Big Bang Theory

Physics Today 70, 40 (2017); 10.1063/PT.3.3427

Hidden worlds of fundamental particles

Physics Today 70, 46 (2017); 10.1063/PT.3.3594

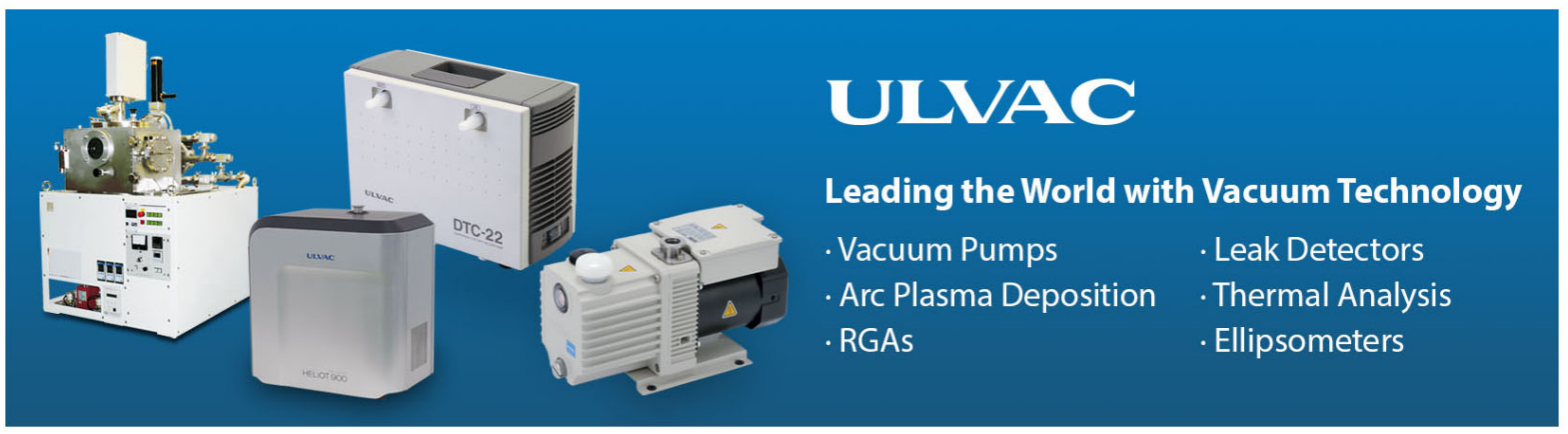


meteorology students, sometimes lose themselves in a welter of computer simulations. Sometimes what is missing is to simply read the sky!"

Anthony J. Sadar Allegheny County Health Department Pittsburgh, Pennsylvania

\section{Conductors, Semiconductors, Superconductors}

\section{An Introduction to Solid State Physics}

\section{Rudolf P. Huebener \\ Springer, 2015. \$59.99 paper \\ (215 pp.). ISBN 978-3-319-09140-2}

With its commonplace title, Conductors, Semiconductors, Superconductors: An Introduction to Solid State Physics might appear to be just another introductory text covering this well-established area of contemporary physics. Actually, it is anything but typical.

Written by University of Tübingen professor Rudolf Huebener and originally published in German in 2013, Conductors, Semiconductors, Superconductors aims to generate interest in students and young scientists and to serve, as stated in the preface, "as a motivating pre-stage and companion of the established and very detailed textbooks." It guides the reader through the major

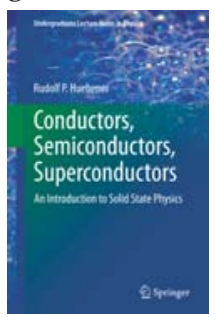
themes of solid-state physics from a historical and human perspective and provides insight into the way the topics were originally understood by early leaders in the field.

Mainstream science textbooks do not usually focus on the human aspects; Huebener's, by contrast, includes lesser-known details about seminal figures. The result is a technical text interlaced with many historical digressions, including portraits, reproductions of lab notebooks, images of various instruments, and historical illustrations of the described phenomena. Consequently, the book provides a refreshing reminder of why, even in our digital age, good old-fashioned record keeping remains important. The text also notes that many important scientific discoveries were made by young scientists, a fact that should motivate the target audience.

The book's unique approach makes it a pleasure to read and will inspire readers to want to learn more about its topics - both their science and their his- tory. The impressive list of selected physics and chemistry Nobel Prizes is a nice finishing touch that highlights the importance of the subjects selected for treatment in this relatively concise text.

The author chose to focus on electronic phenomena that, not being amenable to classical-physics descriptions, demonstrate the reality of quantum mechanics. He describes experimentally accessible macroscopic quantum systems, notably superconductors and systems with long-range magnetic order, from the unified point of view of the electronic structure of solids. And he underscores the important roles of the atomic arrangement in the crystal lattice, the nature of the chemical bond, chemical composition, and behavior at the nanometer scale. The discussion is illustrated by examples of milestone advances and inventions, such as the discovery of superconductivity and the invention of x-ray spectroscopy, transistors, solidstate lasers, electron microscopy, and quantum interference devices.

Huebener is known worldwide for his scientific contributions to many of the phenomena he discusses. His book Magnetic Flux Structures in Superconductors (extended reprint, Springer, 2001) is one of the most cited academic works on that topic. I have no doubt that Conductors, Semiconductors, Superconductors, the most recent of his books targeting younger scientists, will be of broad interest to students and researchers in the natural sciences and engineering. It will also nicely complement more advanced but somewhat dry academic texts. I, for one, would be delighted to recommend this book to the students taking my class.

Ruslan Prozorov Ames Laboratory Ames, Iowa

\section{Are We All Scientific Experts Now?}

\section{Harry Collins \\ Polity, 2014. \$12.95 paper (140 pp.). ISBN 978-0-7456-8204-4}

In his remarkable manifesto, sociologist Harry Collins, a major voice in the field of science studies, answers the provocative question presented in the book's title: Are We All Scientific Experts Now? Collins starts out by outlining science's fall from grace in the public's eye and by presenting a tongue-in-cheek caricature of scientific expertise based on the zeitgeist he holds responsible for the distortions. He goes on to present a compelling "taxonomy of expertise" that leads him to deny, in no uncertain terms, the specialist status to anyone who is not a member of the scientific community and is not able to make "judgements from the platform of the norms and aspirations that drive the community."

Collins contrasts two waves of studies that led to very different views of science. In the first, iconic and heroic scientists with unique knowledge were elevated to almost mythological status; the rest of society was left to marvel at and celebrate the benefits of their work. The second wave, in which Collins played a major role, set out to question and deconstruct the scientific mythos. Its call to arms was Thomas Kuhn's The Structure of Scientific Revolutions (University of Chicago Press, 1962).

Indeed, the second wave showed that science was much more messy and complex than previously thought. That is illustrated by what chemist and philosopher Michael Polanyi called "tacit knowledge," the idea that scientists and other creators are guided by more than what can explicitly be exchanged using the written and spoken word. Tacit knowledge is beautifully illustrated by Collins in his study of the development of the transversely excited atmospheric laser. The realization that science has a tacit, cultural component led some in the public arena to call for an egalitarian-derived dismissal and devaluation of scientific expertise, a diminishing of scientists' authority in society, and an advocacy for broader participation of nonexperts when making technical decisions.

Unfortunately, Collins only briefly sketches the unintended and detrimental impacts that the diminishment of science's role has had on science itself and on Western society. That vaguely criticized zeitgeist warrants a much deeper analysis. At the same time, I'm impressed by the author's self-critical assessment of wave two-in particular, his acknowledgment of the need to restore the privileged role of experts in technical decision making. His call for a third wave of science studies to correct overinterpretations and reverse policies and societal trends caused by the diminished recognition of scientific expertise is in itself remarkable.

Collins's new book contains some gems. In particular, his taxonomy of expertise will help scientists understand why the foundations of expertise often shift and experts are often contested in the public arena. There's ubiquitous expertise, which is acquired simply by growing up in society and learning to 
do such basic things as operate a microwave, remote control, or graphic interface. On the other end of the spectrum is specialist expertise, which requires a decade or more of training, often as an apprentice gaining tacit knowledge through interactions with accomplished practitioners.

One of Collins's biggest contributions to science studies is the concept of interactional expertise. It can be attributed to a person who has obtained a certain level of fluency in a field even if he or she does not directly participate in experiments, theory building, or data analysis. His book Gravity's Shadow: The Search for Gravitational Waves (University of Chicago Press, 2004) is proof that Collins himself has gained interactional expertise by engaging with scientists for more than three decades.

Collins uses Joseph Weber as an example in Are We All Scientific Experts Now? to demonstrate the roles that tacit knowledge, community, and time play when judging mavericks in science. Weber claimed to have seen the first experimental evidence of a gravitational wave, but the community subsequently dismissed his claims, and he became a tragic figure after about 1975. Collins points out that in the current internet age, Weber's papers would be accessible to anyone, not just scientists. However, he adds, being part of a specific scientific culture and community of specialists is the only way to truly contextualize such work - and then have a basis to ignore it. Collins missed an opportunity to contrast the Weber affair with the rather different case of Raymond Davis Jr, whose claim of detecting a solar neutrino flux much lower than that predicted by the standard solar model was challenged for decades even as Davis maintained his credibility in the community. He ultimately received the 2002 Nobel Prize in Physics "for the detection of cosmic neutrinos."

Are We All Scientific Experts Now? makes the important point that when people demand that scientists in such contentious fields as climatology "show their work," it is an act of subterfuge. When scientists do release their data, few elected officials or members of the general public have enough interactional expertise to make a sound judgment. More's the pity: Interactional expertise could be a powerful tool for those in the political arena confronted with bogus scientific claims backed by the testimony of some maverick. Time and the messy scientific process may tell if the maverick scientist is a Weber or a Davis.

We can only hope-as Collins advocates for at the end-that more social scientists will take concrete steps to once again "elevate science to a special position in our society."

Thomas Vogt University of South Carolina Columbia

\section{new books}

\section{biological and medical physics}

Fluorescent Methods for Molecular Motors. C. P. Toseland, N. Fili, eds. Springer, 2014. \$189.00 (298 pp.). ISBN 978-3-03480855-2

Micro- and Nanoengineering of the Cell Surface. J. M. Karp, W. Zhao, eds. William Andrew/Elsevier, 2014. \$199.00 (378 pp.). ISBN 978-1-4557-3146-6

Physics and Biology: From Molecules to Life. J. F. Allemand, P. Desbiolles, eds. World Scientific, 2015. \$58.00 (182 pp.). ISBN 978981-4618-92-2

\section{photodiode preamplifiers}

perfect for pulse detection!
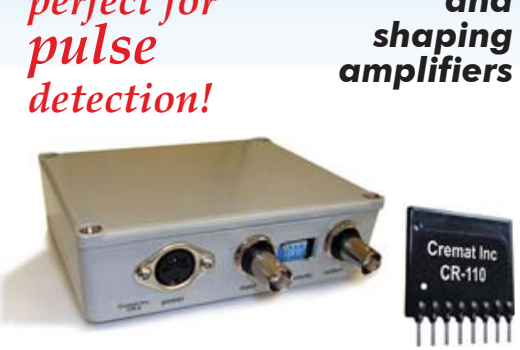

all product specifications can be found online at: http:///cremat.com

Cremat's low noise charge sensitive preamplifiers (CSPs) can be used to read out pulse signals from p-i-n photodiodes, avalanche photodiodes (APDs), SiPM photodiodes, semiconductor radiation detectors (e.g. Si, CdTe, CZT), ionization chambers, proportional counters, surface barrier/PIPS detectors and PMTs.

Our CSPs and shaping amplifiers are small epoxy-sealed plug-in modules less than 1 in 2 in area. We also

provide evaluation

boards for these c r e m a t modules, letting you 950 Watertown St easily and quickly West Newton, MA integrate these parts 02465 USA into your instrumenta- +1(617)527-6590 tion.

info@cremat.com

\section{X-Ray and Gamma Ray Detectors}

\section{- Solid State Design Easy to Use - Low Cost Choose your detector}
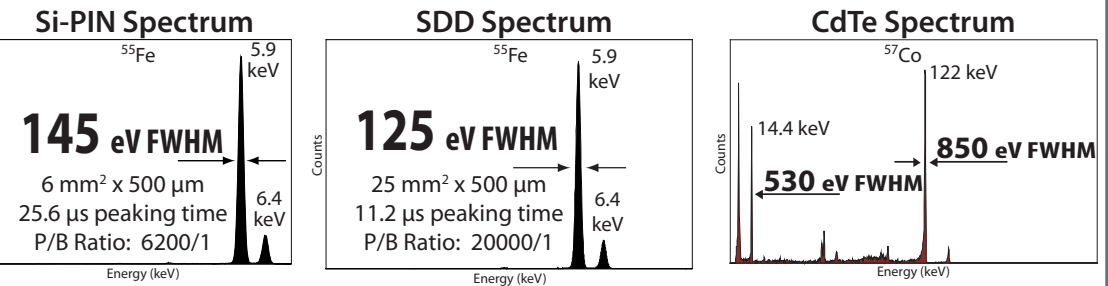

\section{FAST SDD ${ }^{\circledR}$ with Count Rate $>1,000,000$ CPS}

\section{Choose your configuration}

Complete Spectrometer
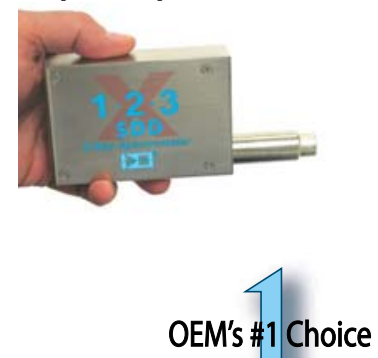

OEM Components
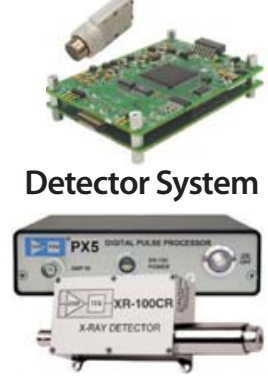

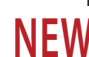

\section{Exp}

Experimenter's Kit

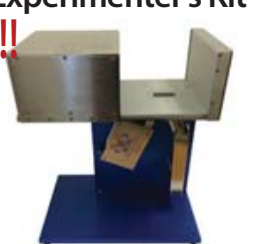

